\title{
The impact of tensioning device mal-positioning on strand tension during Anterior Cruciate Ligament reconstruction
}

\author{
Rajesh Maharjan ${ }^{1 \dagger}$, John J Costi ${ }^{2 \dagger}$, Richard M Stanley ${ }^{2+}$, David Martin ${ }^{3 \dagger}$, Trevor C Hearn ${ }^{1 \dagger}$ and John R Field ${ }^{1 *+}$
}

\begin{abstract}
Background: In order to confer optimal strength and stiffness to the graft in Anterior Cruciate Ligament (ACL) reconstruction, the maintenance of equal strand tension prior to fixation, is desired; positioning of the tensioning device can significantly affect strand tension This study aimed to determine the effect of tensioning device malpositioning on individual strand tension in simulated cadaveric ACL reconstructions.

Methods: Twenty cadaveric specimens, comprising bovine tibia and tendon harvested from sheep, were used to simulate ACL reconstruction with a looped four-strand tendon graft. A proprietary tensioning device was used to tension the graft during tibial component fixation with graft tension recorded using load cells. The effects of the tensioning device at extreme angles, and in various locking states, was evaluated.

Results: Strand tension varied significantly when the tensioning device was held at extreme angles $(p<0.001)$ or in 'locked' configurations of the tensioning device $(p<0.046)$. Tendon position also produced significant effects ( $p$ $<0.016)$ on the resultant strand tension.

Conclusion: An even distribution of tension among individual graft strands is obtained by maintaining the tensioning device in an unlocked state, aligned with the longitudinal axis of the tibial tunnel. If the maintenance of equal strand tension during tibial fixation of grafts is important, close attention must be paid to positioning of the tensioning device in order to optimize the resultant graft tension and, by implication, the strength and stiffness of the graft and ultimately, surgical outcome.
\end{abstract}

\section{Background}

Surgeons increasingly favour reconstruction of the anterior cruciate ligament with the multi-strand tendon autograft in preference to bone-patella tendon-bone grafts (BPTB) because of the relatively low complication rate [1] and availability of improved fixation methods; equally tensioned quadrupled hamstring tendon $(\mathrm{QHT})$ grafts have been shown stronger and stiffer than BPTB grafts [2-4]; Initial graft tension plays a vital role in maintaining joint kinematics and in situ forces in the graft during knee motion $[5,6]$. The application of excessive intraoperative tension can precipitate joint stiffness, the development of abnormal stresses on the articular cartilage

\footnotetext{
* Correspondence: lantbruks@bigpond.com

+ Contributed equally

'Comparative Orthopaedic Research Surgical Facility, School of Medicine,

Flinders University, Bedford Park, 5042, South Australia, Australia

Full list of author information is available at the end of the article
}

and menisci, and which may also interfere with graft revascularization [7-9]. Conversely, inadequate graft tension will lead to excessive joint laxity [3]. To maintain optimum biomechanical properties it appears important to generate, and maintain, similar tension in all four strands of the QHT graft at the time of graft tensioning and tibial fixation [10-12].

Currently, there is no consensus regarding the amount of tension to apply to a graft when it is secured [1]. An initial tension of $44 \mathrm{~N}$ is considered optimum by some, but there is no empirical evidence for this argument $[13,14]$. Restoration of anterior translation to within 3 $\mathrm{mm}$ of the native ACL condition, after cyclic loading, required approximately $68 \mathrm{~N}$ initial tension to be applied [15]. Graft tensioning has been evaluated in numerous cadaveric studies [7,15-19], with considerable variation in graft tension observed between surgeons, prompting the suggestion that graft tension should be more accurately

\section{Biomed Central}


measured and controlled intra-operatively [17]; Gertel et al [20] demonstrated that the direction of tensioning and the flexion angle of the knee at which the tension was applied also plays a significant role in the initial graft tension.

Various techniques have been used to maintain uniform tension in all strands of a QHT graft. Bellemans et al [21] demonstrated the use of one spiked staple to fix the hamstring tendon to the tibia in order to maintain the appropriate tension prior to introduction of an interference screw. Hamner et al [10] produced equal tension in the strands by applying weights. Commercially available tensioning devices can reportedly produce and maintain equal tension in the strands of QHT. In principle, when the tensioning device is pulled it exerts equal tension in all of the strands. However, when the tensioning device is deviated from that axis, which may occur while inserting an interference screw, strand tension may alter. This may have an adverse impact on the biomechanical properties of the graft, which in turn may affect the surgical outcome.

This study aimed to quantify the effects, on individual strand tension and stress, on tensioning device malpositioning. The null hypotheses were as follows:

1. Individual strand tensions, during looped fourstrand tendon graft ACL reconstruction, are equal when using a tensioning device in line with the longitudinal axis of the tibial tunnel.

2. Angulation of the tensioning device, with respect to the long axis of the tibial tunnel, will result in equal strand tension.

3. Locking the tensioning device at extreme angles will result in equal strand tension.

\section{Methods}

Simulation of ACL reconstruction with a looped fourstrand tendon graft was performed using cadaveric bovine tibiae and sheep superficial digital flexor (SDF) tendons harvested from skeletally mature individuals. The utilization of animal-derived tissues was approved by the Institutional Animal Welfare Committee.

To obtain a study power of 0.8 with an alpha of 0.05 , the required sample size was determined to be $n=20$. To this end 20 cadaveric reconstructions were performed and tested.

The ACL Tie Tensioner (Mitek, Johnson and Johnson, USA) was evaluated for its ability to apply reproducible individual strand tension when positioned as might occur in clinical practice (Figure 1).

Retrieved tendon strands were whipstitched using No. 1 braided polyester suture (Ethibond, Ethicon, Inc., USA); Suture loops were attached to hooks connected to each load cell. The diameter of the graft composite was measured by passing it through an incremental

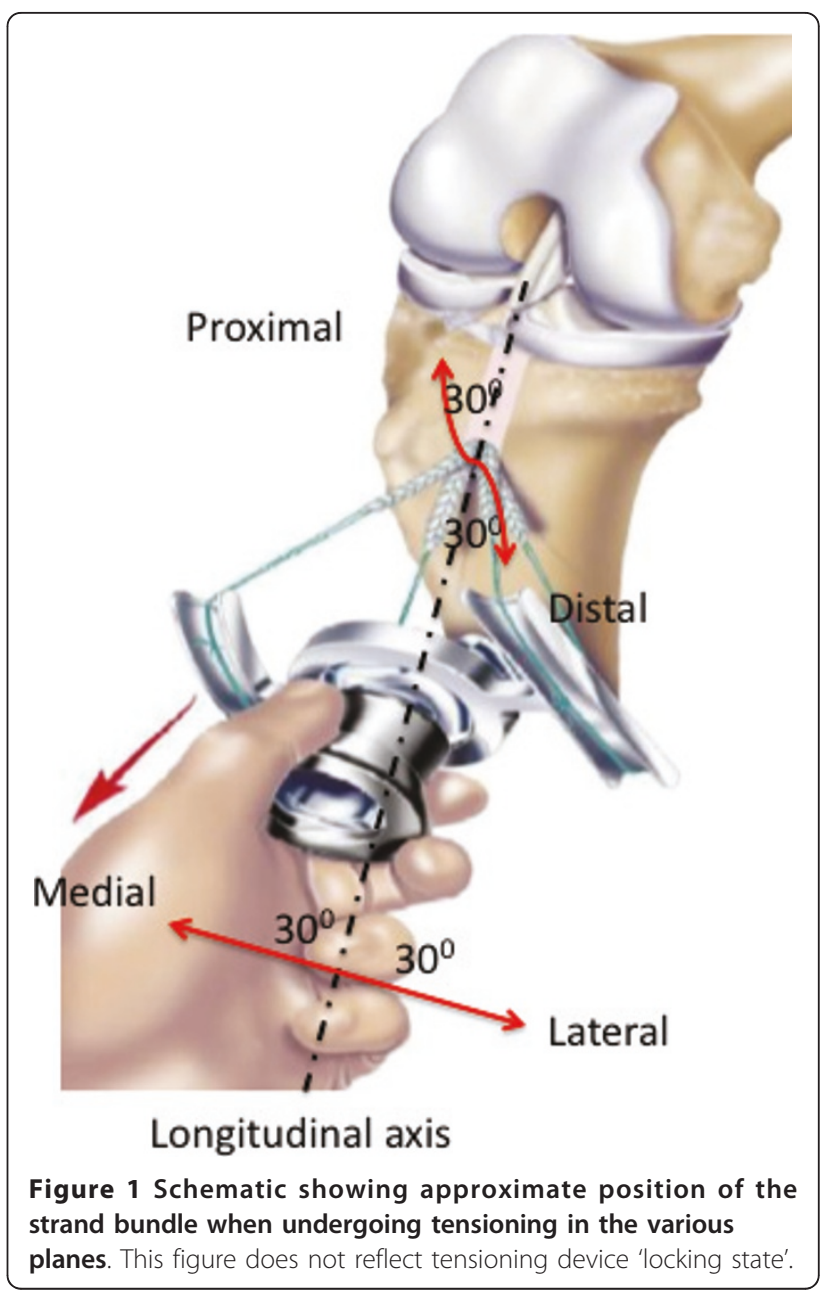

sizing block to achieve a bundled strand diameter of $8.00 \mathrm{~mm}$. The femoral aspect of the graft was stabilized at the level of the tibial plateau using a circular rod passed through the centre of the tendon loops and which rested on the tibial plateau.

Biomechanical tests were performed with an Instron materials testing system (Instron Pty Ltd, High Wycombe, UK). Once placed in the testing system, with the tibial tunnel at zero degrees (vertical), each tendon suture loop was attached to a $25 \mathrm{~kg}(223 \mathrm{~N})$ load cell (AL Design Inc, Buffalo, New York, USA model ALD.75 DIA UTC MINI$50 \mathrm{lb}$ ). All four load cells were then attached to the tensioning device such that each arm supported two tendons and their accompanying load cells (Figure 2). Load cells were then balanced before applying tension to the tendon strands. These were loaded to $150 \mathrm{~N}$ in tension for 10 sinusoidal cycles at $0.1 \mathrm{~Hz}$., allowing the tendons to reach a steady state of hysteresis and reduce the effects of creep and stress relaxation found in viscoelastic tissue. Once completed the Instron was kept in load control to maintain a tension of $150 \mathrm{~N}$ on the tendons. 


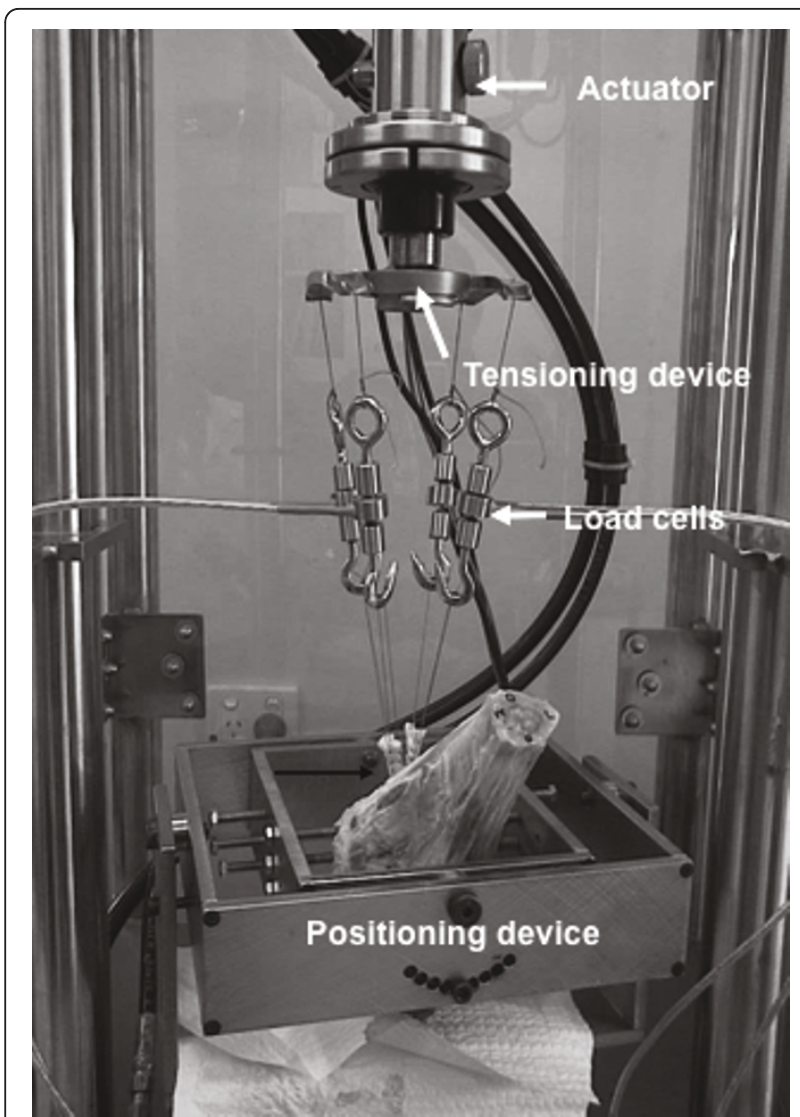

Figure 2 The component arrangement for testing of reconstructions: The tensioning device is positioned in series with the reconstruction, four load cells and the load-train of the Instron as depicted.

The tibia was then moved to place the tunnel at the positions described below. At each position, the Instron load was allowed to return to $150 \mathrm{~N}$. The position was maintained for five seconds before moving to the next position. This allowed time for the tendons to undergo creep recovery from their prior location and which also served as a reference point for the next sequence of tibial tunnel angulations.

The tensioning device was first evaluated in the unlocked position (longitudinal alignment with axis of tibial tunnel) then locked clock wise (CW) followed by counter-clockwise (C-CW) locking (Figure 3). The tests were repeated at each of the seven predetermined positions (tensioning device angle) for each of the locking states.

The load in each tendon strand and actuator displacement, was recorded for subsequent data analysis. Statistical analysis was performed with SPSS (SPSS Inc., Illinois, USA). Repeated measures analysis of variance (ANOVA) was used to evaluate the data. The independent variables, tensioning device state (unlocked, locked

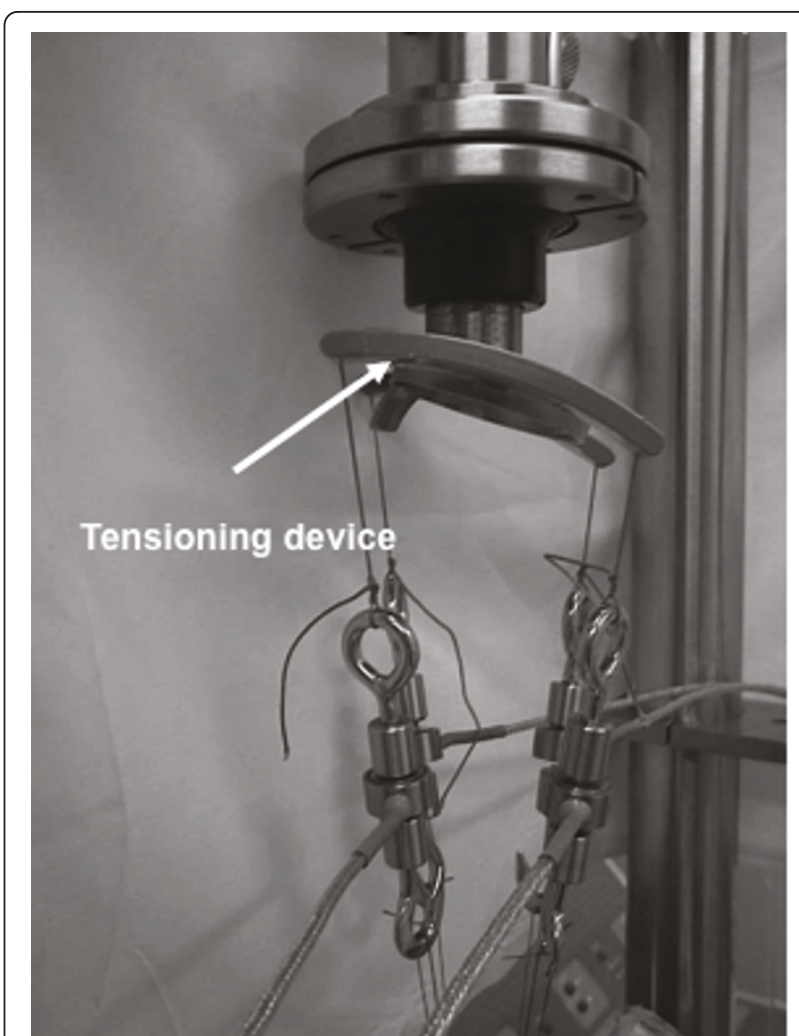

Figure 3 Tensioning device locking state: The arms of the tensioning device are shown in the locked clockwise position with the central ring firmly pressed against the tensioning tube.

clockwise, and locked counterclockwise), tensioning device position (7 positions, 01, D30, P30, 02, M30, L30, 03) and tendon position (4 positions; bottom lateral [BL], top medial [TM], top lateral [TL] and bottom medial $[\mathrm{BM}]$ ) were considered as within-subject factors. The dependant variable was the tension in each strand. For all statistical comparisons, a probability level of $\mathrm{p}<$ 0.05 was considered significant.

\section{Results}

Mean strand tensions for each test are displayed in Table 1 and presented graphically in Figure 4. These provide a synopsis of the strand bundle response to each of the positions adopted and also reflect the 'locking state' of the tensioning device.

When the tensioning device is utilized in the unlocked position (aligned with the longitudinal axis of the tunnel), the angle at which the tensioning device is held produces a significant effect $(p<0.0001)$ on the outcome measures. Conversely, tendon position does not produce a significant effect $(\mathrm{p}=0.051)$. The interaction between tensioning device angle and tendon position is significant $(\mathrm{p}<0.001)$ with BL significantly greater than TM at all angles $(\mathrm{p}<0.025)$. 
Table 1 The data displayed represents the mean strand tension (Newtons) \pm standard deviation compiled from testing each reconstruction $(n=20)$

\begin{tabular}{cccccccc}
\hline Unlocked & $\mathbf{0 1}$ & $\mathbf{D} \mathbf{1 0}$ & $\mathbf{P 3 0}$ & $\mathbf{0 2}$ & $\mathbf{M 3 0}$ & L30 & $\mathbf{0 3}$ \\
\hline Top-medial & $36.3 \pm 1.4$ & $36.7 \pm 2.2$ & $35.6 \pm 1.6$ & $36.1 \pm 1.9$ & $34.2 \pm 1.8$ & $37.7 \pm 2.0$ & $35.4 \pm 1.7$ \\
\hline Top-lateral & $36.9 \pm 1.6$ & $36.9 \pm 2.6$ & $36.1 \pm 2.5$ & $37.1 \pm 1.8$ & $38.5 \pm 2.7$ & $34.6 \pm 2.3$ & $37.6 \pm 1.7$ \\
\hline Bottom-medial & $36.8 \pm 3.1$ & $36.8 \pm 2.7$ & $35.6 \pm 1.6$ & $36.8 \pm 2.8$ & $38.5 \pm 2.9$ & $35.6 \pm 2.4$ & $37.8 \pm 2.3$ \\
\hline Bottom-lateral & $38.1 \pm 3.0$ & $38.3 \pm 2.8$ & $38.8 \pm 2.9$ & $37.8 \pm 2.9$ & $36.7 \pm 2.9$ & $40.0 \pm 2.3$ & $37.2 \pm 2.6$ \\
\hline Locked-clockwise & $\mathbf{0 1}$ & $\mathbf{D} \mathbf{3 0}$ & $\mathbf{P 3 0}$ & $\mathbf{0 2}$ & $\mathbf{M 3 0}$ & $\mathbf{L 3 0}$ & $\mathbf{0 3}$ \\
\hline Top-medial & $39.6 \pm 1.4$ & $35.6 \pm 3.2$ & $38.4 \pm 2.1$ & $39.6 \pm 1.6$ & $35.8 \pm 2.4$ & $39.5 \pm 2.2$ & $39.0 \pm 1.5$ \\
\hline Top-lateral & $40.6 \pm 2.2$ & $35.9 \pm 3.3$ & $38.6 \pm 3.7$ & $40.9 \pm 1.3$ & $40.7 \pm 2.9$ & $36.1 \pm 3.3$ & $41.3 \pm 1.3$ \\
\hline Bottom-medial & $33.7 \pm 3.0$ & $38.7 \pm 5.2$ & $34.8 \pm 3.2$ & $33.6 \pm 2.5$ & $37.7 \pm 3.1$ & $33.1 \pm 2.2$ & $34.4 \pm 2.3$ \\
\hline Bottom-lateral & $34.3 \pm 3.1$ & $39.2 \pm 3.0$ & $35.8 \pm 3.5$ & $33.8 \pm 2.7$ & $33.4 \pm 3.0$ & $39.5 \pm 3.4$ & $33.5 \pm 2.8$ \\
\hline Locked-counterclockwise & $\mathbf{0 1}$ & $\mathbf{D 3 0}$ & $\mathbf{P 3 0}$ & $\mathbf{0 2}$ & $\mathbf{M 3 0}$ & $\mathbf{L 3 0}$ & $\mathbf{0 3}$ \\
\hline Top-medial & $33.9 \pm 1.9$ & $33.4 \pm 2.2$ & $33.4 \pm 2.2$ & $33.7 \pm 1.8$ & $32.1 \pm 2.5$ & $34.9 \pm 2.1$ & $33.3 \pm 1.6$ \\
\hline Top-lateral & $34.7 \pm 1.9$ & $33.3 \pm 2.4$ & $33.5 \pm 3.6$ & $35.0 \pm 1.4$ & $35.6 \pm 2.6$ & $32.1 \pm 2.8$ & $35.1 \pm 1.5$ \\
\hline Bottom-medial & $39.5 \pm 2.9$ & $40.2 \pm 3.3$ & $39.6 \pm 2.3$ & $39.6 \pm 2.7$ & $41.9 \pm 2.8$ & $38.2 \pm 2.3$ & $40.2 \pm 2.3$ \\
\hline Bottom-lateral & $40.4 \pm 2.6$ & $42.2 \pm 2.9$ & $41.5 \pm 3.7$ & $40.0 \pm 2.1$ & $38.8 \pm 3.6$ & $43.5 \pm 2.1$ & $39.8 \pm 2.5$ \\
\hline
\end{tabular}

Testing was performed with the tensioning device held in three locking states (unlocked, locked-clockwise and locked-counterclockwise. Individual strand response to loading (top-medial, top-lateral, bottom medial and bottom lateral) are recorded at each position (01, 02, 03: neutral; D30, P30: distal or proximal excursion; M30, L30: lateral or medial excursion).

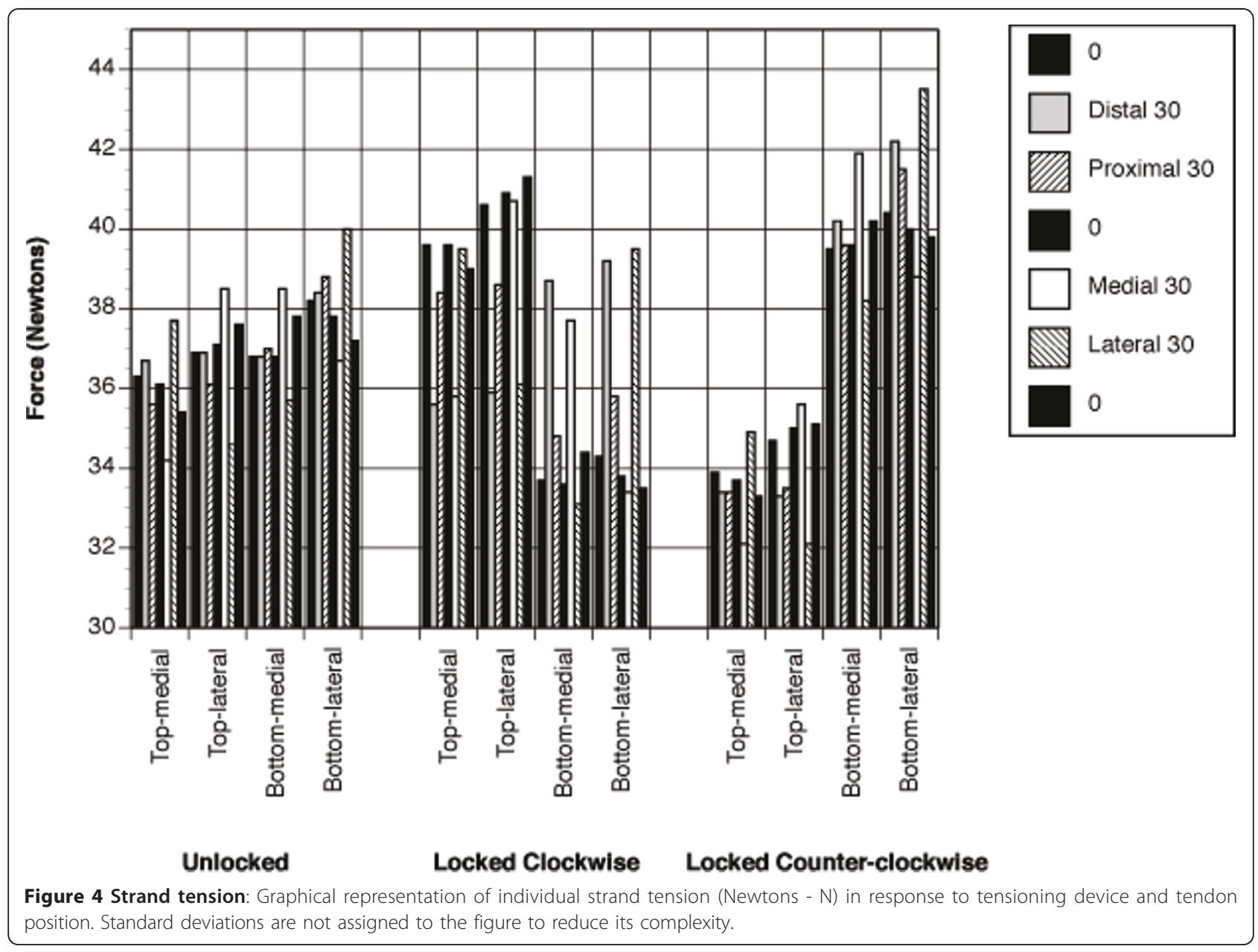


The results of tensioning device locking produced significant main effects with tensioning device angle $(\mathrm{p}<$ $0.001)$, locking state $((\mathrm{p}<0.046)$ and tendon position $(\mathrm{p}<0.016)$ all producing significant effects on the resultant strand tension. The interaction between tensioning device angle and tendon position was significant ( $\mathrm{p}<$ $0.001)$ as was locking state and tendon position $((\mathrm{p}<$ $0.001)$ with the interaction between locking state, tensioning device angle and tendon position also producing significant effects $(\mathrm{p}<0.001)$

\section{Discussion}

ACL reconstruction with the looped four-strand tendon graft has gained popularity. Although clinical outcomes $[4,15,17,18]$ are similar to BPTB grafting there appear to be fewer complications with optimal fixation techniques now available $[13,14]$. The distribution of tension in all strands of the graft, an integral factor in its success, is gaining widespread attention [15,18]. Due to the composite nature of the graft, it appears essential to apply equal tension to all the strands during tibial fixation $[8,15]$. This, it is suggested, will provide optimal strength and stiffness to the graft leading to a better surgical outcome $[5,6,22]$. It is further proposed that any disparity in the tension between strands may lead to disproportionate tensile loading and which, may ultimately lead to early rupture of the strands, weakening the entire reconstruction.

Brown et al [23] evaluated the manual application of tension to grafts followed by fixation with $4.5 \mathrm{~mm}$ cortical screws in combination with plastic, spiked washers. In order to produce equal tension in all four strands, suture loops were created from the graft ends; no data was presented to confirm equality in strand tension. Hammer et al [10 ], produced equal tension in strands by applying known weights. This study showed that when strands were clamped, they exhibited better tensile properties. The mean maximum load obtained for four strand grafts was $2831 \pm 538 \mathrm{~N}$ when the tension had been applied manually and $4590 \pm 674 \mathrm{~N}$ when it had been applied with a weight. However, tension in individual strands was not documented.

The objective in performing this cadaveric study was to quantify the level of tension applied to all strands of a looped four-strand tendon graft before tibial fixation. This was undertaken to investigate the impact of malpositioning of the tensioning device on the resultant strand tension. The analysis was conducted at three neutral positions $(01,02$, and 03 ) and with the tensioning device helf in various positions (medial and lateral excursion - $30^{\circ}$; proximal and distal excursion $-30^{\circ}$ ) and locking states (Figures 1, 2, and 3).

Our first null hypothesis was shown, in part, to be correct; strand tensions were not significantly different when the tensioning device was at the 01 and 02 neutral positions. However, strand tension differed significantly at the third neutral position, 03 (Figure 4). One possible explanation was that this position (03) followed mediolateral excursion of the tensioning device which may have indiced residual tendon deformation, altering their biomechanical behavior.

Our second null hypothesis evaluated the effect of extreme angulation of the tensioning device, when deviated to $30^{\circ}$ from the neutral position in all four planes, in the unlocked state (Figure 4). Strand tensions were recorded at four positions; distal 30 (D30), proximal 30 (P30), medial 30 (M30) and lateral 30 (L30). Minimal variations in strand tension were observed when data was recorded with either proximal or distal excursion of the strands. A possible interpretation is that at D30 and P30 the tendons are deviated proximally and distally from their longitudinal axis, which may have reduced impact on changes to their biomechanical properties. The plane of proximal-distal rotation lies closer to the longitudinal axis of the tunnel and hence the tendons.

Conversely, strand tension showed a significant differences when the tensioning device was deviated to M30 and L30 allowing rejection of the second null hypothesis in this specific situation. A possible explanation is that at M30 and L30 there is medio-lateral excursion of the tendons away from their longitudinal axis. Such a variation, in the direction of load application, may result in significant structural deformation of the tendons, which in turn will have a tangible impact on their biomechanical behavior.

We rejected our third null hypothesis in that locking state of the tensioning device produced a significant impact on strand tension (Figure 4). The locked counter-clockwise state showed a greater significant difference to its other locked counterpart. A possible reason could be the shifting of the body of the tensioning device in relation to its arms, as occurs during locking; this may impact on the direction of tension transmission during tensioning. In the unlocked state, the body of the device is positioned centrally between the arms. This arrangement may contribute to a more uniform distribution of strand tension. However, when the device is locked, the body of the device moves in proximity to either the proximal or distal end of the arms, depending on the locking state. Hence, in the clockwise direction, with the tendons situated proximally, TM and TL may experience greater tensile force (39.6 and $40.6 \mathrm{~N}$ ) as the arms of the device move away from them. In distally positioned tendons, BM and $\mathrm{BL}$, may be subject to lesser tension (33.7 and $34.2 \mathrm{~N}$ ) as the arms move towards them. Their alignment with the tunnel axis was altered, and they were displaced distally by the moving arms. Thus, locking the device causes significant variation in 
strand tension, which may influence the biomechanical behavior of the graft strands.

Although the strand tensions in the unlocked state were uniformly distributed, stresses were not equal because strand cross-sectional areas were different. Strand crosssectional area had a significant impact on the resultant stress generated within the strand. Inequality of stresses may lead to early rupture of the smaller strands as they bear the greater tension per unit area. A possible solution may be the harvest of tendons having similar crosssectional area. This would allow distribution of stresses more uniformly across all strands, ultimately providing a more optimal mechanical environment for the composite graft.

Their remains no agreement regarding the quantification of tissue viscoelasticity nor reliable modelling [24]; difficulty arises in the delineation of viscoelastic and pre-conditioning effects, as both are manifest by similar response features. The efficacy of anterior cruciate ligament reconstruction, using either QHT or BPTB grafts is thought to depend on the relative amounts of graft elongation or creep; hysteresis and creep effects appear highest during the first few loading cycles with more than 160 cycles required to reach a steady state, beyond which there was no further creep and hysteresis almost constant $[25,26]$. It appears that the effect of cyclic preconditioning is the progressive recruitment of fibres $[23,26]$.

In the current study we have arbitrarily chosen to allow a 5 second period of relaxation between tests; this may lead to conjecture regarding our experimental methodology and possible impact of creep on the resultant data. It has been shown [27] that contraction duration significantly affects tendon strain at all levels of applied force. In response to these findings it is appropriate, in order to compare tendon mechanical properties, that the duration of loading be standardized as it has been in the current study.

A recent study [28], further complicates the situation with the suggestion that equal-stress tensioning may provide an alternative to equal-tension tensioning as performed in the current study; data derived suggested that equal-stress tensioning of tendon grafts resisted graft creep significantly better, raising the issue of the utilization of graft material having equal cross sectional areas.

\section{Conclusion}

The findings of this study provide useful information for ACL reconstructive surgery, in which a looped fourstrand tendon graft is utilized. It appears, that the optimal position to induce and maintain uniform strand tension, with a tensioning device, is along the longitudinal axis of the tibial tunnel. Any deviation from this axis, more so in the medial and lateral planes, appears to result in a significant variation in strand tension. Similarly, superior strand tension was obtained by maintaining the tensioning device in an unlocked state.

This study is a simulation of the human surgical procedure for graft tensioning. The reconstructions performed in this study, using animal-tissue, do not therefore provide a completely analogous system for comparison. However, it does appear that surgeons should consider closer attention to optimal alignment of tensioning devices in use; if this is done, a more uniform distribution of forces may be generated in the four loop components of the QHT reconstruction providing augmented mechanical characteristics of the reconstruction and, by implication, possibly improve graft longevity and effectiveness.

\section{Acknowledgements}

The authors wish to acknowledge the contribution of David Carney, Johnson and Johnson, Adelaide, Australia for his, and his company's support of this study

\section{Author details}

${ }^{1}$ Comparative Orthopaedic Research Surgical Facility, School of Medicine, Flinders University, Bedford Park, 5042, South Australia, Australia. ${ }^{2}$ Flinders Medical Devices and Technologies - Biomechanics and Implants Group, School of Computer Science, Engineering and Mathematics, Flinders University, South Australia, Australia. ${ }^{3}$ Sportsmed, Stepney, South Australia, Australia.

\section{Authors' contributions}

Authors contributed variably to the concept, design and performance of this study. All authors have read and approved the final manuscript.

\section{Competing interests}

The authors declare that they have no competing interests.

Received: 10 January 2010 Accepted: 28 June 2011

Published: 28 June 2011

\section{References}

1. Amis AA, Jacob RP: Anterior cruciate ligament graft positioning, tensioning and twisting. Knee Surg sports Traumatol Arthros 1998, 6:s2-s12.

2. Corry IS, Webb JM, Clingeleffer AJ, et al: Arthroscopic reconstruction of the anterior cruciate ligament. A comparison of patellar tendon autograft and four-strand hamstring tendon autograft. Am J Sports Med 1999, 27:444-454.

3. Yasuda $K$, Ichiyama $H$, Kondo $E$, et al: An in vivo biomechanical study on the tension-versus-knee flexion angle curves of two grafts in anatomic double-bundle anterior cruciate ligament reconstruction. Arthroscopy 2007, 23(8):869-876.

4. Wagner M, Kaab MJ, Schallock J, et al: Hamstring tendon versus patella tendon anterior cruicate ligament reconstruction using biodegradable interference fit fixation: a prospective matched-group analysis. Am J Sports Med 2005, 33(9):1327-1336.

5. Samuelsson K, Andersson D, Jarlsson J: Treatment of anterior cruciate ligament injuries with special reference to graft type and surgical technique: an assessment of randomized controlled trials. Arthroscopy 2009, 25(10):1139-1174.

6. Arneja S, McConkey MO, Mulpuri K, et al: Graft tensioning in anterior cruciate ligament reconstruction: a systematic review of randomized controlled trials. Arthroscopy 2009, 25(2):200-207.

7. Mae T, Shino K, Nakata K, et al: Optimization of graft fixation at the time of anterior cruciate ligament reconstruction. Part i: effect of initial tension. Am J Sports Med 2008, 36(6):1087-10093. 
8. Figueroa D, Calvo R, Vaisman A, et al: Effect of tendon tensioning: an in vitro study in porcine extensor tendons. Knee 2010, 17(3):245-248.

9. Scheffler SU, Schmidt T, Gangey I, et al: Fresh-frozen free-tendon allografts versus autografts in anterior ligament reconstruction: delayed remodelling and inferior mechanical function during long-term healing in sheep. Arthroscopy 2008, 24(4):448-458.

10. Hammer $\mathrm{DL}$, Brown $\mathrm{CH}$, Steiner ME, et al: Hamstring tendon grafts for reconstruction of the anterior cruciate ligament: Biomechanical evaluation of the use of multiple strands and tensioning techniques. J Bone Joint Surg Am 1999, 81-A(4):549-557.

11. Grover DM, Howell SM, Hull ML: Early tension loss in an anterior cruciate ligament graft. A cadaver study of four tibial fixation devices. J bone Joint Surg Am 2005, 87-A(2):381-390.

12. Nurmi JT, Kannus $P$, Sievanen $H$, et al: Interference screw fixation of soft tissue grafts in anterior cruciate ligament reconstruction: Part 2; effect of preconditioning on graft tension during and after screw insertion. Am J Sports Med 2004, 32(2):418-424.

13. Fu FH, Bennett $\mathrm{CH}$, Lattermann $\mathrm{C}:$ Current trends in Anterior cruciate ligament reconstruction-Part 1. Am J Sports Med 1999, 27(6):821-830.

14. Fu FH, Bennett $\mathrm{CH}, \mathrm{Ma} \mathrm{CB}$, Menetrey J, Lattermann C: Current trends in Anterior cruciate ligament reconstruction-Part 11. Am J Sports Med 2000, 28(1):124-136.

15. Boylan $D$, Greis PE, West $J R$, et al: Effects of initial graft tension on knee stability after $\mathrm{ACL}$ reconstruction using hamstring tension: A cadaveric study. Arthroscopy 2003, 19(7):700-705.

16. Chungfu C, Noorani S, Vercillo F, Woo SL: Tension patterns of the anteromedial and postero-lateral grafts in double-bundle anterior cruciate ligament reconstruction. J Orthop Res 2009, 27(7):879-884.

17. Cunningham R, West JR, Greis PE, Burks RT: A survey of the tension applied to a doubled hamstring tendon graft for reconstruction of the anterior cruicate ligament. Arthroscopy 2002, 18(9):983-988.

18. Fleming BC, Brady MF, Bradley MP, Banerjee R, Hulstyn MJ, Fadale PD: Tibiofemoral compression force differences using laxity and force based initial graft tensioning techniques in the anterior cruciate ligamentreconstructed cadaveric knee. Arthroscopy 2008, 24(9):1052-1060.

19. Hoshino Y, Kuroda R, Nagamune K, Nishimoto K, Yagi M, Mizuno K Yoshiya S, Kurosaka M: The effect of graft tensioning in anatomic 2bundle $\mathrm{ACL}$ reconstruction on knee joint kinematics. Knee Surg Sports Traumatol Arthrosc 2007, 15(5):508-514.

20. Gertel TH, Lew WD, Lewis JL, et al: Effect of anterior cruciate ligament graft tensioning, direction, magnitude, and flexion angel on knee biomechanics. Am J Sports Med 1993, 21(4):572-580.

21. Bellemans J, Eid T, Fabry G: A modified technique for tibial interference screw fixation of hamstring anterior cruciate ligament grafts. Arthroscopy 1999, 15(6):669-671.

22. Labs K, Perka C, Schneider F: The biological and biomechanical effect of different graft tensioning in anterior cruciate ligament reconstruction: An experimental study. Arch Orthop Trauma Surg 2002, 122(4):193-199.

23. Brown $\mathrm{CH}$, Hammer D, Hecker AT, et al: Biomechanics of semitendinosus and gracilis tendon grafts. Sports Medicine, Stockholm 1995, 31-40.

24. Einat $R$, Yoram $L$ : Recruitment viscoelasticity of the tendon. J Biomech Eng 2009, 131(11):111-118

25. Simonian PT, Levine RE, Wright TM, et al: Response of hamstring and patellar tendon grafts for anterior cruciate ligament reconstruction during cyclic tensile loading. Am J Knee Surg 2000, 13(1):8-12.

26. Schatzmann L, Brunner P, Staubli HU: Effect of cyclic preconsitioning on the tensile properties of human quadriceps tendons and patellar ligaments. Knee Surg Sports Traumatol Arthrosc 1998, 6(Suppl 1):S56-61.

27. Pearson SJ, Burgess K, Onambele GN: Creep and the in vivo assessment of human patellar tendon mechanical properties. Clin Biomech 2007, 22(6):712-717.

28. Conner CS, Morris RP, Vallurupalli S, et al: Tensioning of anterior cruciate ligament hamstring grafts: comparing equal tension versus equal stress. Arthroscopy 2008, 24(12):1323-1329.

doi:10.1186/1749-799X-6-33

Cite this article as: Maharjan et al:: The impact of tensioning device mal-positioning on strand tension during Anterior Cruciate Ligament reconstruction. Journal of Orthopaedic Surgery and Research 2011 6:33.

\section{Submit your next manuscript to BioMed Central and take full advantage of:}

- Convenient online submission

- Thorough peer review

- No space constraints or color figure charges

- Immediate publication on acceptance

- Inclusion in PubMed, CAS, Scopus and Google Scholar

- Research which is freely available for redistribution

Submit your manuscript at www.biomedcentral.com/submit 